\title{
Theroratical Study of Fully Printed Magnetically Tunable Reconfigurable Patch Antenna
}

\author{
Farhan Abdul Ghaffar ${ }^{1,2}$, Mohammad Vaseem ${ }^{1}$, Langis Roy ${ }^{2}$, Atif Shamim ${ }^{1}$ \\ ${ }^{1}$ King Abdullah University of Science and Technology (KAUST), IMPACT Lab, Computer, Electrical and Mathematical Sciences and \\ Engineering (CEMSE) Division \\ Thuwal 23955-6900, Kingdom of Saudi Arabia \\ ${ }^{2}$ Faulty of Electrical and Applied Science at University of Ontario Institute of Technology (UOIT) \\ Ontario, Canada \\ farhan.ghaffar@kaust.edu.sa,mohammad.vaseem@kaust.edu.sa, langis.roy@uoit.ca, atif.shamim@kaust.edu.sa
}

\begin{abstract}
Due to varying wireless standards in different regions, there is a big desire to have reconfigurable and tunable components which can be tuned to the frequency of interest. At present a single wireless device, such as a mobile phone contains multiple antennas, filters, all of them working at different frequencies. It will be great if an antenna or a filter can be tuned to work at different frequencies, thus the overall number of antennas or filters in a device can be reduced. This control is possible through a magnetic field, if these components are built on a magnetic substrate. Traditional magnetic substrates are expensive that involve complicated fabrication process and operate the material in saturated state making them inefficient. A better approach would be to operate the antenna or any other microwave device in the partially magnetized state. In this work, the authors present the theory and design of a polarization reconfigurable patch antenna with frequency tunablity in the unsaturated state. Operating away from the saturated state can improve the overall efficiency of the RF components. The analytical model is initially verified using a high frequency simulator to predict the antenna performance on a magnetic substrate with normal bias. A low-cost printing technique where the substrate could be realized using magnetic ink is used for the implementation of the antenna. A circular patch antenna working at $6 \mathrm{GHz}$ is used for the validation of the theory. When triggered by an external magnetic field, the as-fabricated antenna shows a maximum tuning range of $\sim 16 \%$ and $\sim 5 \%$ for the two splitted frequency points. A good match is achieved between the theory, simulations and measurements of the antenna.
\end{abstract}

Keywords - antenna, iron oxide, inkjet-printing, magnetic material, reconfigurability,

\section{INTRODUCTION}

In the wireless industry, there is a rising demand in using radio frequency $(\mathrm{RF})$ components that can be tuned to operate at different frequency band so as to provide secure and efficient communication. In addition, the new applications require antenna diversity that can be achieved by radiation pattern reconfigurability. The utilization of a reconfigurable antenna in such systems can effectively decrease the cost, complexity of the design and reduce the size of the system. The tunablity and reconfigurability of the antennas can be realized by several methods. Such as, ferrites have been popularly used by RF engineers to demonstrate the antenna versatility. Many phase shifters, phase scanning arrays and tunable antennas based on ferrites have been reported in literature [1-2]. However, all of these reported designs operate the substrate in the saturated state which makes them unpopular due to the stringent requirements of the modern wireless communication systems.

Recently, the authors have demonstrated ferrite Low Temperature Co-fired Ceramic (LTCC) technology as a substitute to the bulk ferrite substrates [3-6]. The results thus achieved show that how ferrite technology is still integrable in the current RF systems. Even with all the benefits accompanying the LTCC based designs it cannot be neglected that the technology is quite expensive and has some limitations in terms of microwave performance of the components. Thus, there is a need of a multilayer technology that is cost effective and can compete with the LTCC based designs in terms of figure of merit.

In this work, we adopt an additive manufacturing process for a fully printed magnetically tunable antenna. Fabrication of magnetic substrate is performed with custom magnetic ironoxide nanoparticles ink. However, antenna is realized using silver-organo-complex (SOC) based metallic ink. This technology is proposed as an alternate option to the already existing LTCC technology. In addition, the authors propose a theoretical model to predict the impedance and radiation performance of the fully printed antenna in the partially magnetized state. Although, this analytical model was initially reported by Pozar in 1992 [7], it did not discuss the operation in the partially magnetized state. Here, the antenna is operated in the partially magnetized state. The as-fabricated antenna shows polarization reconfigurability and frequency splitting, when triggered by an external magnetic field, which is well matched with the theory and simulations of the antenna in the partially magnetized state.

\section{THEORY}

For the theoretical analysis, a circular patch antenna, as shown in Fig. 1, has been studied on a partially magnetized ferrite substrate. The radius of the antenna is labelled as ' $a$ '. Due to the structure of the antenna, cylindrical coordinate system will be used to evaluate the fields inside the antenna structure. The theoretical analysis is based on the cavity model of the patch antenna.

The substrate is considered to be biased in the $\mathrm{Z}$ direction which results in the permeability tensor of (1). The elements of the tensor (i.e. $\mu, \kappa, \mu_{\mathrm{z}}$ ) are defined in [6] and where $\mu_{0}$ is the free space permeability. 


$$
\mu=\mu_{0}\left[\begin{array}{ccc}
\mu & -j \kappa & 0 \\
j \kappa & \mu & 0 \\
0 & 0 & \mu_{z}
\end{array}\right]
$$

Using the permeability tensor, Ampere's and Faraday's laws are applied on the partially magnetized patch antenna for $\mathrm{TM}^{\mathrm{Z}}$ mode.

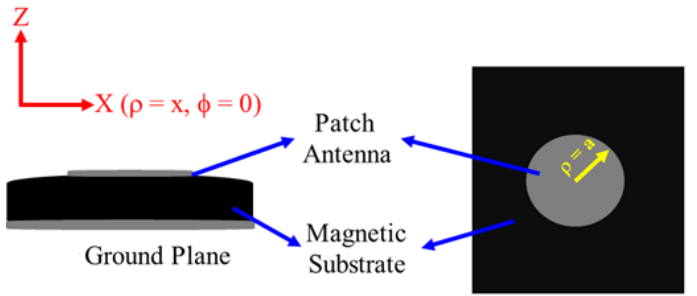

Fig. 1. Stack up and the geometry of a circular patch antenna on a magnetic substrate

Application of Ampere's Law for dominant $\mathrm{TM}_{11}$ mode results in (4).

$$
\frac{1}{\rho} \frac{\partial\left(\rho h_{\phi}\right)}{\partial z}-\frac{1}{\rho} \frac{\partial\left(h_{\rho}\right)}{\partial z}=j \omega \varepsilon e_{z}
$$

where $\frac{\partial}{\partial z}=0$ which causes the two electric field components i.e. $e_{\rho}$ and $e_{\phi}$ to be 0. Applying Faraday's Law using the permeability tensor for the $\mathrm{Z}$ direction of bias provides the following:

$$
\begin{gathered}
\frac{1}{\rho} \frac{\partial\left(e_{z}\right)}{\partial \phi}=j \omega \mu h_{\rho}-\omega \kappa h_{\phi} \\
\frac{1}{\rho} \frac{\partial\left(e_{z}\right)}{\partial \rho}=j \omega \mu h_{\phi}+\omega \kappa h_{\rho}
\end{gathered}
$$

Simplifying (3.1) and (3.2) together gives rise to the following wave equation:

$$
\nabla^{2} e_{\mathrm{z}}+k^{2} e_{\mathrm{z}}=0
$$

where,

$$
\begin{gathered}
k=\omega \sqrt{\mu_{e} \varepsilon} \\
\mu_{e}=\frac{\mu^{2}-\kappa^{2}}{\mu} \\
\nabla^{2}={\frac{\partial}{\partial \rho^{2}}}^{2}+\frac{1}{\rho} \frac{\partial}{\partial \rho}+\frac{1}{\rho^{2}} \frac{\partial^{2}}{\partial \phi^{2}}
\end{gathered}
$$

In the above equation $k$ is the wave number and $\mu_{\mathrm{e}}$ is the permeability for the extraordinary (E) mode of propagation in ferrites [7]. The solution of (4) yields the following electric field inside the patch structure.

$$
e_{z}=\left\{A J_{1}(k \rho)+B Y_{1}(k \rho)\right\}\left(C e^{j \phi}+D e^{-j \phi}\right)
$$

where $J_{1}$ and $Y_{1}$ are Bessel functions of the first kind, first order and the second kind, first order, respectively. $A, B, C$ and $D$ are the coefficients for the functions used in the above solution. Since $Y_{1}$ is infinity for $\rho=0$ i.e. the origin (antenna is centered at origin), therefore (8) is reduced further by using $B=0$. Now the electric field component $e_{\mathrm{z}}$ can be used to compute the magnetic field component. Here it is important to concentrate on $h_{\phi}$, since the boundary condition at $\rho=a$, enforces $h_{\phi}$ to go to zero. This is because the walls of the patch antenna substrate are assumed to be perfect magnetic conductors. Therefore, $h_{\phi}$ is given by (9).

$$
\begin{aligned}
h_{\phi} \frac{j \kappa}{\omega \mu \mu_{e}}\left\{\frac{1}{\rho} j A J_{1}(k \rho)\right. & \left(C e^{j \phi}-D e^{-j \phi}\right)- \\
& \left.\left(-j \frac{\mu}{\kappa}\right) A J_{1}{ }^{\prime}(k \rho)\left(C e^{j \phi}+D e^{-j \phi}\right)\right\}
\end{aligned}
$$

Enforcing the boundary condition on $h_{\phi}=0$ at $\rho=$ a, results in the following solution.

$$
J_{1}{ }^{\prime}(k a) \pm \frac{\kappa}{a \mu} J_{1}(k a)=0
$$

(10) is the closed form solution to find out the resonant frequency of the antenna using its roots and is similar to the findings of Pozar in [7]. However, the contribution of this work is to incorporate the partial magnetized regime into the theoretical analysis. The equation shows that when a ferrite or a magnetic medium is biased normally, it causes a circular patch antenna to resonate at two different resonant frequencies with two different senses of circular polarization. This will be covered in the results section.

\section{MATERIALS AND FABRICATION}

Magnetic iron oxide $\left(\mathrm{Fe}_{3} \mathrm{O}_{4}\right)$ is prepared in the form of spherical nanoparticles $(\sim 20 \mathrm{~nm})$ by solution process. The asprepared iron oxide nanoparticles are then treated with oleic acid to make it compatible with SU8 2000 (UV-curable epoxy resin from Microchem). Further, oleic acid treated magnetic nanoparticles are mixed with SU8 in 50:50 weight \% ratios for formulating screen-printable ink. When the ink was ready, it was printed using squeegee (a manual screen-printing technique). The detailed information on materials, inkformulation and steps for the fabrication process of free standing magnetic substrate are provided in our previous report [8-9]. The circular patch (CP) antenna is ink-jet printed with eight layers each of SOC ink on top of magnetic substrate and also on bottom for ground plane, as shown in Fig. 2.

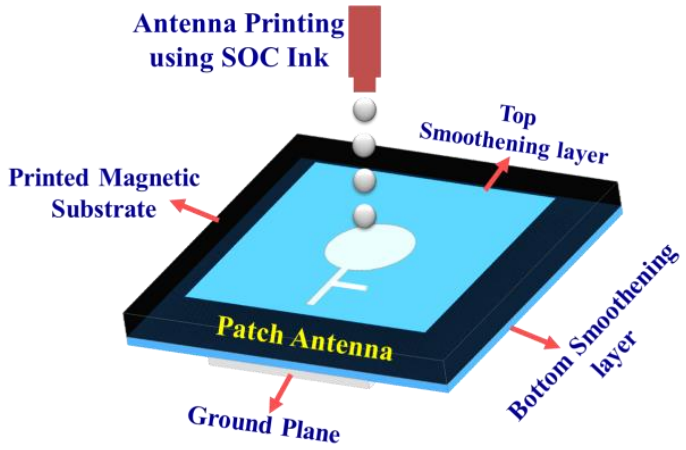

Fig. 2. 3D depiction of as-design magnetic substrate with printed antenna 
The SOC ink is cured and sintered with infrared (IR) heating for five minutes (after every single layer of printing). The antenna is optimized in Ansys HFSS with using the magnetic and dielectric properties of the material to operate at $6 \mathrm{GHz}$. The optimized printed antenna has a circular radius of $\sim 4100 \mu \mathrm{m}$ on a magnetic substrate (thickness of $\sim 1500 \mu \mathrm{m}$ ). The open circuit stub is printed with a length of $\sim 3500 \mu \mathrm{m}$ which is $\sim 1600 \mu \mathrm{m}$ away from the edge of the antenna. The final fabricated prototype is shown in Fig. 3.

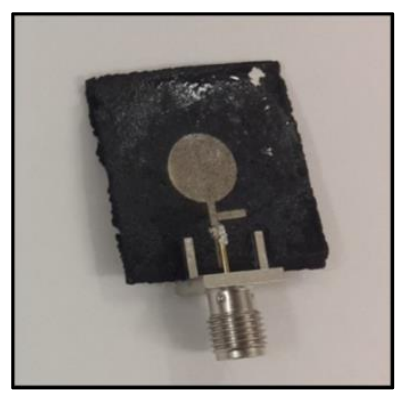

Fig. 3. Fabricated Prototype

\section{ANTENNA RESUlts}

To verify the theoretical model the antenna is initially simulated using CST Microwave Studio. Like theory, the simulations also show that in the presence of the bias the antenna works at two different resonant frequencies with circular polarization. The results of the theoretical and simulation model will be discussed in conjunction with the measured results. The fabricated antenna is initially measured without any bias where $6 \mathrm{GHz}$ is seen to be the antenna center

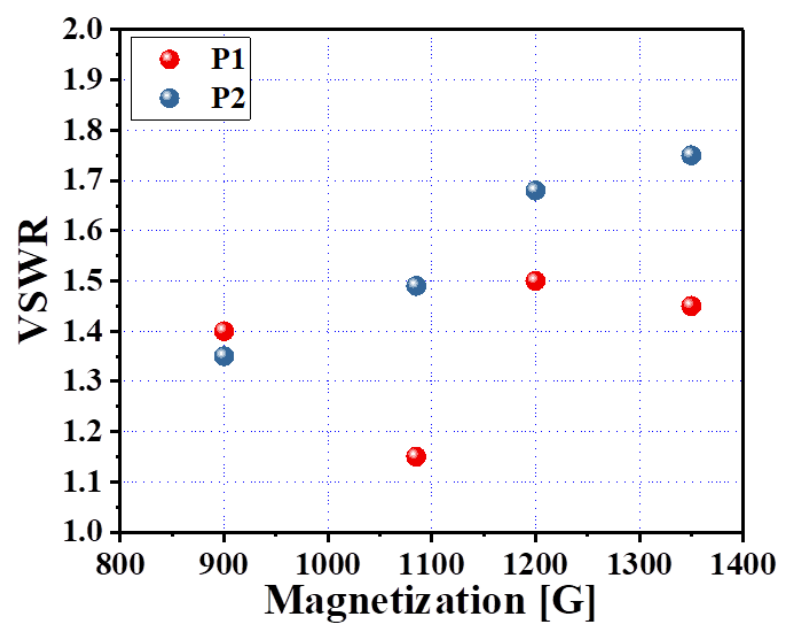

Fig. 4. Measured VSWR vs magnetization for different bias strengths

frequency. An electromagnet is used to provide the variable magnetic field. The antenna plane is kept parallel to the poles of the electromagnet thus ensuring normal magnetic bias. It is observed that under biased conditions the reflection coefficient of the antenna show two different frequencies with decent matching as expected. In other words, the biasing of the antenna causes it to resonate at two frequency points unlike the unbiased condition. Fig. 4 shows the matching of the antenna in the biased state at the two new resonant frequencies for different bias strengths. It can be seen that the antenna maintains its matching when the bias is applied. The VSWR of the antenna for any bias value does not exceed 2. Thus, it can be concluded that the antenna maintains a good matching condition.

It is shown in Fig. 5 that as the bias is increased, the two frequency points deviates from each other. Thus, Fig. 5 confirmed a good agreement between the theory, simulation and measurement results which confirms the operation of the antenna in the partially magnetized state. It is recognized that the two frequencies have circular polarization with opposite sense of rotation which is shown in theory and simulations. These results have been verified through measurements of the realized antenna. As the direction of the bias, each frequency point can exhibit LHCP or RHCP. For $+Z$ direction of bias, P1 is found to be LHCP while P2 is RHCP. On the other hand, for $-\mathrm{Z}$ direction, the bias is reversed so the two polarizations will interchange. Thus using the magnetic bias the linearly polarized antenna can be operated as a circularly polarized (CP) antenna.

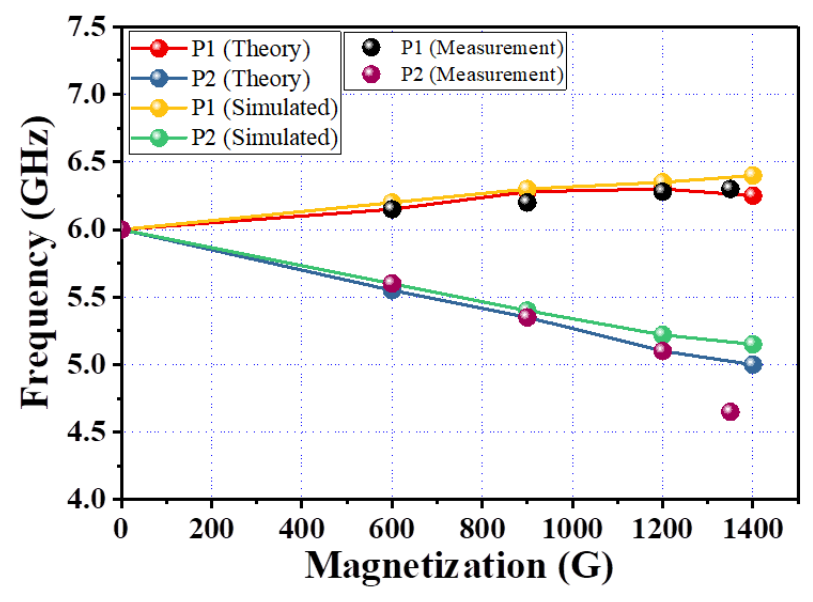

Fig. 5. Theory, simulations and measurement for frequency splitting of the circular patch antenna due to the applied magnetostatic bias

\section{CONCUlSION}

In this report, we presented theory and design of a circular patch antenna with polarization reconfigurable and frequency tunablity on a partially magnetized substrate. Fabrication of magnetic substrate is performed with custom magnetic ironoxide nanoparticles ink. However, antenna is realized using SOC based metallic ink. The realized antenna is used to validate the tuning of the antenna which is predicted by the theory and simulations. The antenna shows a measured tuning range of $\sim 5 \%$ and $\sim 16 \%$ for the two polarizations. The antenna results show the viability of the new magnetic ink and fabrication process for the design of many reconfigurable microwave components.

\section{REFERENCES}

[1] D. M. Pozar, et. al., "Magnetic tuning of a microstrip antenna on a ferrite substrate", Elect. Lett., vol. 24, no. 12, pp. 729-731, 1988.

[2] A. Petosa, et. al., "Magnetically tunable ferrite resonator antenna", Elect. Lett., vol. 30, no. 13, pp. 1021-1022, 1994.

[3] E. Arabi, et. al., "Tunable bandpass filter based on partially magnetized ferrite LTCC with embedded windings for SoP applications," IEEE Micro. Wless. Comp. Lett., vol. 25, no. 1, pp. 16-18, 2015. 
[4] F. A. Ghaffar, et. al., "A Partially Magnetized Ferrite LTCC Based SIW Phase Shifter for Phased Array Applications", IEEE Transactions on Magnetics, vol. 51, 4003108, 2015.

[5] F. A. Ghaffar, et. al., "A Ferrite LTCC Based Dual Purpose Helical Antenna Providing Bias for Tunability," IEEE Antennas and Wireless Propagation Letters, vol. 14, 831, 2015.

[6] F. A. Ghaffar, et. al., "Theory and design of a tunable antenna on a partially magnetized ferrite LTCC substrate", IEEE Transactions on Antennas and Propagation, vol. 62, no. 3, pp. 1238-1245, 2014.

[7] D. M. Pozar, "Radiation and scattering characteristics of microstrip antennas on normally biased ferrite substrates," IEEE Transactions on Antennas and Propagation, vol. 40, no. 9, pp. 1084-1092, 1992.
[8] F. A. Ghaffar, et. al., "A Fully Printed Ferrite Nano-Particle Ink Based Tunable Antenna", IEEE International Symposium on Antennas and Propagation, AP-S/URSI, 2016.

[9] M. Vaseem, et. al., "Iron Oxide Nanoparticle-Based Magnetic Ink Development for Fully Printed Tunable Radio-Frequency Devices," Adv. Mater. Technol. vol. 3, pp. 1700242, 2018. 